\title{
Preparation of polyamide 6/silica nanocomposites from silica surface initiated ring-opening anionic polymerization
}

\author{
M. Yang, Y. Gao, J. P. He, H. M. Li* \\ Institute of Polymer Science, Chemistry College, Xiangtan University, Xiangtan 411105, Hunan Province, P. R. China
}

Received 20 March 2007; accepted in revised form 27 May 2007

\begin{abstract}
Polyamide 6/silica nanocomposites were synthesized by in situ ring-opening anionic polymerization of $\varepsilon$-caprolactam in the presence of sodium caprolactamate as a catalyst and caprolactam-functionalized silica as an initiator. The initiator precursor, isocyanate-functionalized silica, was prepared by directly reacting commercial silica with excess toluene 2,4-diisocyanate. This polymerization was found to occur in a highly efficient manner at relatively low reaction temperature $\left(170^{\circ} \mathrm{C}\right)$ and short reaction times $(6 \mathrm{~h})$. FTIR spectroscopy was utilized to follow the introduction and consumption of isocyanate groups on the silica surface. Thermogravimetric analysis indicated that the polyamide 6 was successfully grown from the silica surface. Transmission electron microscopy was utilized to image polymer-functionalized silica, showing fine dispersion of silica particles and their size ranging from 20 to $40 \mathrm{~nm}$.
\end{abstract}

Keywords: nanocomposites, silicas, polyamides, ring-opening polymerization, TEM

\section{Introduction}

In recent years, the preparation of organic/inorganic hybrid materials composed of organic polymers and silica nanoparticles has been widely investigated. The combination of organic polymer components with nanometer-sized silica fillers in a single material has extraordinary significance for the development of hybrid materials with unique properties, for example, improved mechanical and thermal properties $[1,2]$, gas permeability [3-5] and fire retardance [6] compared to the pristine polymer. However, these enhanced properties can only be achieved by adequately dispersing the silica nanoparticles within the polymer matrix.

Generally, physical mixtures of organic polymers and performed silica nanoparticles may lead to separation in discrete phases, resulting in poor mechanical and optical properties. In addition, nanoparticles agglomeration attends to weaken these properties of the resulting materials [7]. An effective approach to overcome these barriers is to functionalize the silica surface with polymers that are identical or structurally similar to the matrix polymers. Among the widely pursued functionalization strategies is the 'grafting from' method $[8$, 9], in which monomers or initiators are first attached to the silica surface to serve as starting points for propagation. For example, the silicabound azo moieties have been used in the conventional radical polymerization to graft vinyl polymers onto silica surfaces $[10,11]$. In addition, silica-bound trichloroacetyl groups combined with $\mathrm{Mo}(\mathrm{CO})_{6}$ were also used to initiate radical graft polymerization of vinyl monomers to give the corresponding polymer-grafted silica [12]. Although the conventional radical graft polymerization approach promises high graft densities, control over polymer molecular weight and architecture can be difficult to achieve. Conversely, the use of living/controlled radical polymerization can result in the grafted polymers with well-defined structure.

*Corresponding author, e-mail: lihuaming@xtu.edu.cn

(C) BME-PT and GTE 
For example, grafting of well-defined polymers from silica particle surfaces was successfully achieved by atom transfer radical polymerization (ATRP) [13]. Furthermore, preparation of the welldefined organic/inorganic nanocomposites via reverse ATRP [14] and in situ radical transfer addition polymerization was also reported [15]. However, these methods are limited only to attachment vinyl polymers to the silica surfaces.

Polyamide 6 (PA6) is a semicrystalline polymer that exhibits excellent chemical stability and mechanical strength properties, and is also competitively priced in comparison to other polyamides [16]. Exceptional value and performance in many products are thus making PA6 the material of choice for a number of consumer goods and industrial applications. Therefore, there has been much interest in nanocomposites of PA6 with inorganic nanoparticles. Recently, Li et al. [17] reported the preparation of $\mathrm{PA} 6 / \mathrm{SiO}_{2}$ nanocomposites from in situ polymerization of $\varepsilon$-caprolactam together with silica nanoparticles. Although homogeneous dispersion of the silica particles in PA6 matrix was found, no covalent linkages between the organic and inorganic domains led to the decreasing of the thermal stability of the nanocomposites.

Here, toluene 2,4-diisocyanate (TDI) was utilized as a surface modifier for silica nanoparticles. The TDI modified silica particles were used as initiator precursor to conduct ring-opening anionic polymerization of $\varepsilon$-caprolactam in the presence of a sodium caprolactamate catalyst. This approach introduced covalent bonds between PA6 and silica particles and consequently overcoming the drawbacks found in the previously reported literatures. In addition, the morphology and the crystallization behavior of the nanocomposites are examined by transmission electron microcopy (TEM) and differential scanning calorimetry (DSC), respectively.

\section{Experimental}

\subsection{Materials}

The porous nano- $\mathrm{SiO}_{2}$ (DP1) with an average primary particle size of $20 \pm 5 \mathrm{~nm}$ and a specific surface area of $700 \pm 30 \mathrm{~m}^{2} / \mathrm{g}$ was purchased from Zhoushan Mingri Nanomaterials Co. Ltd., China. The hydroxyl group concentration was around $15 \mathrm{wt} \%$ as determined by Karl-Fischer titration. The nanoparticles were pre-treated at $140^{\circ} \mathrm{C}$ under vacuum for $48 \mathrm{~h}$ before used. $\varepsilon$-caprolactam was recrystallized from acetone and the crystals were dried at $40^{\circ} \mathrm{C}$ under reduced pressure for a week before use. TDI was used as received. Toluene was dried with sodium and distilled. All other chemicals were used as received.

\subsection{Characterizations}

FTIR spectra were recorded on a Perkin-Elmer Spectrum One spectrometer from 4000 to $500 \mathrm{~cm}^{-1}$ with a $4 \mathrm{~cm}^{-1}$ resolution. Thermal analysis was performed using a TA Q10 instrument under nitrogen atmosphere. For all samples, the following procedure was used: samples were heated at $250^{\circ} \mathrm{C}$ for $5 \mathrm{~min}$ in order to eliminate the influence of thermal history and the effect of heat treatment on the crystalline structure of the materials, then cooled down to $0^{\circ} \mathrm{C}$ to record the crystallization temperature, and then reheated to $250^{\circ} \mathrm{C}$, all at a rate of $20^{\circ} \mathrm{C} / \mathrm{min}$. The recorded temperatures were calibrated using Indium as standard. Thermogravimetric analysis (TGA) was carried out on a WRT-3P instrument with a heating rate of $5^{\circ} \mathrm{C} / \mathrm{min}$ in flowing nitrogen. TEM analysis was performed using a JEM 3010 electron microscope operating at an acceleration voltage of $120 \mathrm{kV}$ with samples prepared by placing a single drop of the formic acid dispersion onto a holey carbon-coated copper grid. The samples were vacuum-dried for $24 \mathrm{~h}$ at room temperature before the measurement. The viscosity-average molecular weights of the polymers are determined at $25^{\circ} \mathrm{C}$ in formic acid using Ubbelohde viscometer.

\subsection{Preparation of isocyanate-functionalized silica $\left(\mathrm{SiO}_{2}-\mathrm{NCO}\right)$}

$5.00 \mathrm{~g}$ of silica (44.1 mmol of hydroxyl group) was dispersed in $200 \mathrm{ml}$ of TDI (1.41 mol) under stirring and the functionalization was undertaken in a dry nitrogen atmosphere at $80^{\circ} \mathrm{C}$ for $72 \mathrm{~h}$. The functionalized silica was recovered as slurry after centrifugating at $10000 \mathrm{rpm}$ for $30 \mathrm{~min}$. The slurry was washed with anhydrous toluene several times to completely remove the unreacted TDI. The slurry was dried in vacuum at $40^{\circ} \mathrm{C}$ for $48 \mathrm{~h}$. 


\subsection{Determination the content of isocyanate groups of the $\mathrm{SiO}_{2}-\mathrm{NCO}$}

The content of isocyanate groups of the $\mathrm{SiO}_{2}-\mathrm{NCO}$ was determined by titration [18]. In a typical experiment, $200 \mathrm{mg}$ of $\mathrm{SiO}_{2}-\mathrm{NCO}$ sample and $20 \mathrm{ml}$ of $0.1 \mathrm{~mol} / \mathrm{l}$ di-n-butylamine in toluene were charged into a flask and the mixture was stirred at room temperature for $1 \mathrm{~h}$. The unreacted di-n-butylamine was backtitrated with $0.1 \mathrm{~mol} / \mathrm{l} \mathrm{HCl}$. The end of titration was controlled by $\mathrm{pH}$. The content of isocyanate groups was calculated by the Equation (1):

isocyanate group $[$ mmol/g $]=0.1\left(V_{0}-V_{1}\right) \frac{M}{W}$

where $V_{0}[\mathrm{ml}]$ is the titer of $0.1 \mathrm{~mol} / 1 \mathrm{HCl}$ for blank, $V_{1}[\mathrm{ml}]$ the titer of $0.1 \mathrm{~mol} / \mathrm{l} \mathrm{HCl}$ for the sample, $M$ the factor of $0.1 \mathrm{~mol} / \mathrm{l} \mathrm{HCl}$, and $W[\mathrm{~g}]$ the weight of the sample.

\subsection{Preparation of caprolactam-functionalized silica}

In a typical experiment, isocyanate functionalized silica (500 mg, $0.919 \mathrm{mmol}$ of isocyanate group), $\varepsilon$-caprolactam (1.04 g, $9.20 \mathrm{mmol}$ ), and toluene $(100 \mathrm{ml})$ were added to a flask equipped with a reflux condenser and a magnetic stir bar. The flask was evacuated and backfilled with nitrogen three times and then left under nitrogen. Subsequently, the mixture was placed in an oil bath and stirred vigorously at $110^{\circ} \mathrm{C}$ for $5 \mathrm{~h}$. After cooling to room temperature, the resulting suspension was diluted with chloroform $(100 \mathrm{ml})$ and filtered. The collected solid was washed with chloroform to com- pletely remove $\varepsilon$-caprolactam. The product was dried in a vacuum oven $\left(55^{\circ} \mathrm{C}\right)$ for $72 \mathrm{~h}$.

\subsection{Preparation of $P A 6 /$ silica nanocomposites}

In a typical experiment, caprolactam-functionalized silica (200 mg, $0.306 \mathrm{mmol}$ of caprolactam), $\varepsilon$-caprolactam $(3.12 \mathrm{~g}, 27.5 \mathrm{mmol})$, and sodium $(42.2 \mathrm{mg}, 1.83 \mathrm{mmol})$ were added to a flask equipped with a reflux condenser and a magnetic stir bar. The flask was evacuated and backfilled with nitrogen three times and then left under nitrogen. Subsequently, the flask was preheated at $80^{\circ} \mathrm{C}$ and sonicated at this temperature for $30 \mathrm{~min}$. The mixture was then transferred to an oil bath and held at $170^{\circ} \mathrm{C}$ for $6 \mathrm{~h}$, in which the initiator, sodium caprolactamate, formed in situ through the reaction of sodium and $\varepsilon$-caprolactam. The resulting solid was dissolved in formic acid $(100 \mathrm{ml})$, precipitated in water $(1000 \mathrm{ml})$, and filtered. The collected solid was washed thoroughly with methanol and then dried under vacuum overnight.

\section{Results and discussion}

In these studies, the porous nano- $\mathrm{SiO}_{2}$ with an average primary particle size of $20 \pm 5 \mathrm{~nm}$ and a specific surface area of $700 \pm 30 \mathrm{~m}^{2} \cdot \mathrm{g}^{-1}$ was purchased from Zhoushan Mingri Nanomaterials Co. Ltd., China. The hydroxyl group concentration was around $15 \mathrm{wt} \%$. The nanoparticles were pre-treated at $140^{\circ} \mathrm{C}$ under vacuum for $48 \mathrm{~h}$ to eliminate possible absorbed water on the surface of the particles. The dried silica was subsequently reacted with TDI to produce isocyanate-functionalized silica $\left(\mathrm{SiO}_{2}\right.$ NCO) (1) (Figure 1). This chemistry allows for

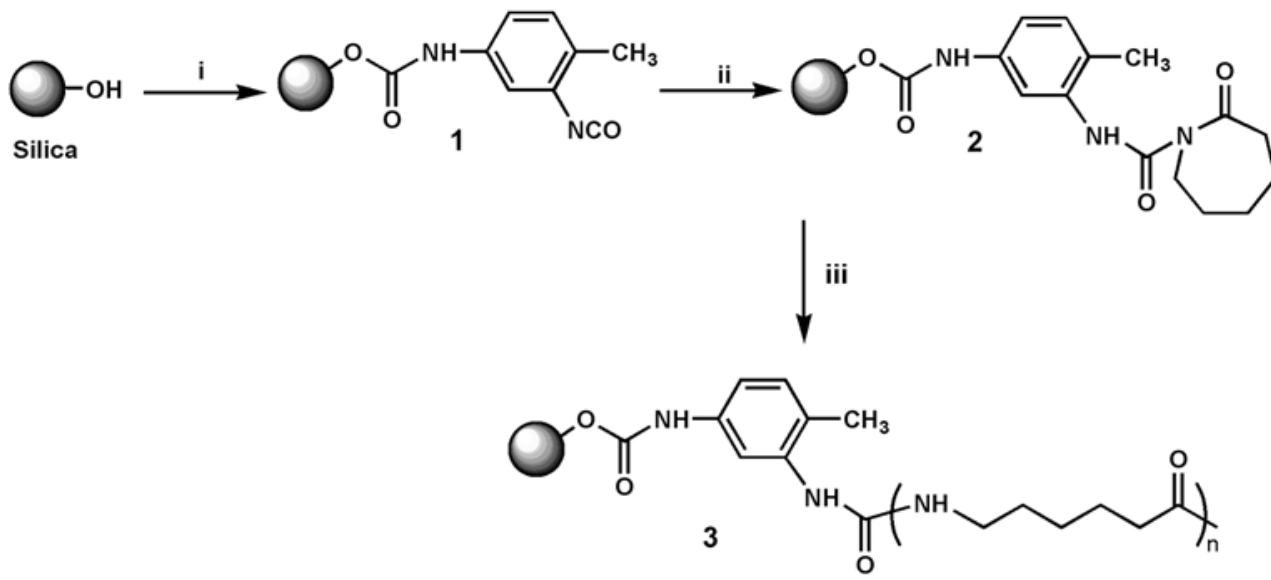

Figure 1. Synthesis of PA6/silica nanocomposites (i) toluene 2,4-diisocyanate, $80^{\circ} \mathrm{C}$; (ii) $\varepsilon$-caprolactam, toluene, $110^{\circ} \mathrm{C}$; (iii) $\varepsilon$-caprolactam, sodium, $170^{\circ} \mathrm{C}$ 
preferential reaction between the para-isocyanate group and the hydroxyl groups on the silica surface, leaving the ortho-isocyanate group intact due to steric hindrance within the TDI molecule $[19,20]$. In a typical experiment, $5.00 \mathrm{~g}(44.1 \mathrm{mmol}$ hydroxyl group) of silica was reacted with $200 \mathrm{ml}$ of TDI $(1.41 \mathrm{~mol})$ at $80^{\circ} \mathrm{C}$. Excess TDI was used as both a solvent to disperse silica and reactant in order to drive the reactions to completion, and was easily removed after each reaction by centrifugation and prolonged washing with anhydrous toluene.

The $\mathrm{SiO}_{2}-\mathrm{NCO}$ (1) was used as initiator precursor to conduct ring-opening anionic polymerization of $\varepsilon$-caprolactam in the presence of a sodium caprolactamate catalyst. The formation of the acyl caprolactam initiator (2) was achieved by reaction between the $\mathrm{SiO}_{2}-\mathrm{NCO}$ and $\varepsilon$-caprolactam. The second step involved silica-bound acyl caprolactam initiated 'activated monomer polymerization' [21, 22]. Considering that the isocyanate functionalities are attached to the silica surface, isocyanate dimerization or trimerization reactions were not observed $[23,24]$, allowing the polymerizations to proceed smoothly. All polymerizations were performed at $170^{\circ} \mathrm{C}$ with variable feed ratio of monomer to silica initiator $\left(R_{\text {feed }}\right)$ as well as reaction time, yielding PA6/silica nanocomposites with different silica contents as depicted in Table 1.

The first and simplest qualitative test to determine whether the 'grafting from' polymerization procedure was successful involved checking the dispersing properties of the products in formic acid (a good solvent for PA6). As expected, on the addition of formic acid to binary blend of free PA6 and silica, the PA6 was completely dissolved and silica was separated out as particles precipitating at the

Table 1. The composition of all composites prepared. Conditions, [initiator]:[sodium $]=1: 6[\mathrm{~mol} / \mathrm{mol}]$; temperature, $170^{\circ} \mathrm{C}$

\begin{tabular}{|c|c|c|c|}
\hline Run & $\begin{array}{c}\text { Polymerization } \\
\text { time [h] }\end{array}$ & $\begin{array}{c}\mathbf{R}_{\text {feed }} \\
\text { [molar ratio] }\end{array}$ & $\begin{array}{c}\text { Content of SiO } 2 \\
\text { [wt\%] }\end{array}$ \\
\hline 1 & 2 & 90 & 13 \\
\hline 2 & 4 & 90 & 9 \\
\hline 3 & 5 & 90 & 7 \\
\hline 4 & 6 & 90 & 5 \\
\hline 5 & 10 & 90 & 4 \\
\hline 6 & 6 & 25 & 14 \\
\hline 7 & 6 & 35 & 10 \\
\hline 8 & 6 & 175 & 2 \\
\hline 9 & 6 & 220 & \\
\hline
\end{tabular}

bottom of the vial. However, when formic acid was added to the ring-opening anionic polymerization product, stable suspension was obtained. This suspension can remain stable for a period of at least 2 weeks, suggesting the formation of covalent linkage between silica and PA6, which did not allow the separation of the two phases. This phenomenon could be attributed to the steric hindrance formed by the grafting of PA6 chains onto nano-sized silica particles, which in turn kept the particles from aggregation and reduce their number of accessible sites [25, 26].

FTIR spectroscopic analysis provided additional evidence that the silica surface reaction proceeded as illustrated in Figure 1. Figure 2 shows the FTIR spectra of pristine silica (spectrum A), $\mathrm{SiO}_{2}-\mathrm{NCO}$ (1) (spectrum B), caprolactam-functionalized silica (2) (spectrum C), and the PA6/silica nanocomposites (3) (spectrum D). The FTIR spectrum of unmodified silica is relatively simple and well assigned [27]. The strong absorbance at $1099 \mathrm{~cm}^{-1}$ is attributed to the $\mathrm{Si}-\mathrm{O}-\mathrm{Si}$ stretch of silica, the absorbances at 1631 and $3434 \mathrm{~cm}^{-1}$ are assigned to the surface hydroxyl groups of silica (Figure 2, spectrum A). Addition of excess TDI to the silica resulted in the incorporation of isocyanate functionalities on the surface of the silica. This was evidenced by the appearance of a clearly discernible band at $2276 \mathrm{~cm}^{-1}$ corresponding to asymmetric stretching of the appended terminal isocyanate groups, and the appearance of an aromatic $\mathrm{C}-\mathrm{C}$ stretch at $1602 \mathrm{~cm}^{-1}$ in the FTIR spectrum B. Additionally, signals corresponding to the $\mathrm{C}=\mathrm{O}$ and

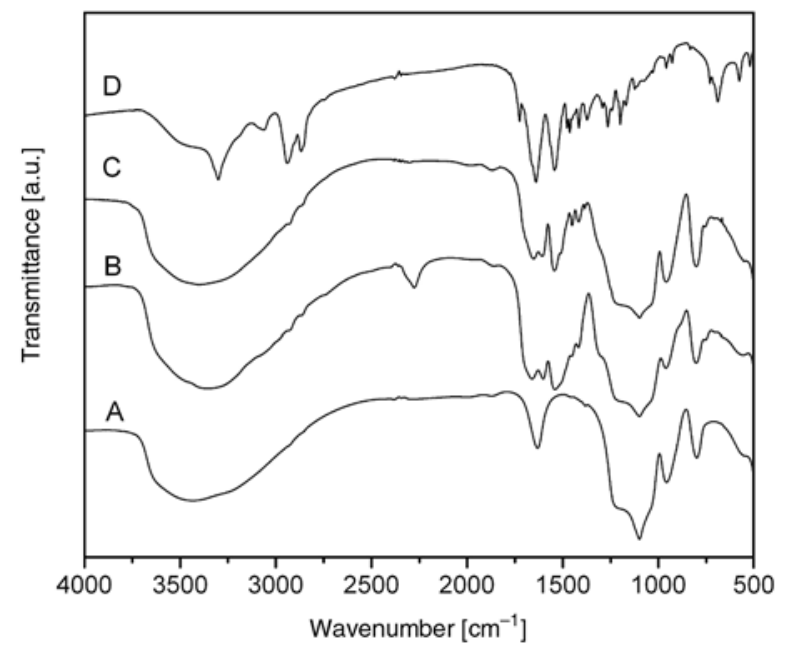

Figure 2. FTIR spectra of (A) pristine silica, (B) $\mathrm{SiO}_{2}-\mathrm{NCO},(\mathrm{C})$ caprolactam-functionalized silicas, and (D) polyamide 6/silica nanocomposites 
$\mathrm{C}-\mathrm{N}$ stretches of the formed carbamate linkages between the silica and the isocyanate functionality in compound (1) can also be seen at $1660 \mathrm{~cm}^{-1}$ and $1220 \mathrm{~cm}^{-1}$, respectively. The surface isocyanate functionalities could then be treated with $\varepsilon$-caprolactam in anhydrous toluene at $110^{\circ} \mathrm{C}$ to introduce the initiating species on the surface of the silica particles (Figure 1). This functionalization reaction was again followed by FTIR spectroscopy to monitor the appearance of $\mathrm{C}-\mathrm{H}$ stretches at 2930 and $2860 \mathrm{~cm}^{-1}$ from the alkyl portions of the attached $\varepsilon$-caprolactam, and the appearance of a more strong $\mathrm{C}=\mathrm{O}$ stretch at $1651 \mathrm{~cm}^{-1}$ and a relatively strong $\mathrm{C}-\mathrm{N}$ stretch at $1265 \mathrm{~cm}^{-1}$ arising from the carbamido linkages (Figure 2, spectrum C). Although the isocyanate signal at $2276 \mathrm{~cm}^{-1}$ is weak, the spectra clearly indicate that the isocyanate signal is not present prior to reaction with TDI or after the addition to $\varepsilon$-caprolactam. Compared with the FTIR spectrum of $\mathrm{SiO}_{2}-\mathrm{NCO}$, the disappearance of the isocyanate stretch after the addition to $\varepsilon$-caprolactam indicates that most of the isocyanates must have been consumed during this reaction, though the low intensity of this FTIR absorption leaves some uncertainty. In the FTIR spectrum of the anonic polymerization product (spectrum $\mathrm{D}$ ), the broad but slightly less intense band at $1543 \mathrm{~cm}^{-1}$ is assigned to a combination of the bending of the $\mathrm{N}-\mathrm{H}$ bond and the stretching of the $\mathrm{C}-\mathrm{N}$ bond of the amide group. The broad band around $3400 \mathrm{~cm}^{-1}$ is due to the hydrogen-bonded amide groups of the PA6, whereas the $\mathrm{C}-\mathrm{H}$ stretching vibrations occur at 2868 and $2942 \mathrm{~cm}^{-1}$. The FTIR results verify that the PA6 chains are covalent attached to the silica nanoparticles.

TGA analysis was performed on the reaction products as depicted in Figure 3. For comparison, the TGA plot of pristine silica is also shown in Figure 3 , and indicates a gradual mass loss of around $10 \mathrm{wt} \%$ as the temperature reaches $700^{\circ} \mathrm{C}$. On the other hand, there is a distinct mass loss region between 200 and $600^{\circ} \mathrm{C}$ for $\mathrm{SiO}_{2}-\mathrm{NCO}$, caprolactam-functionalized silicas, and PA6/silica nanocomposites with different polymer weight proportions. However, it has to be pointed out that part of the weight loss of the silica particles is due to the continued condensation reaction and associated water loss [28]. If the weight retention of the residue at $700^{\circ} \mathrm{C}$ is used as the reference, the weight retention of $\mathrm{SiO}_{2}-\mathrm{NCO}$ at $700^{\circ} \mathrm{C}$ is about

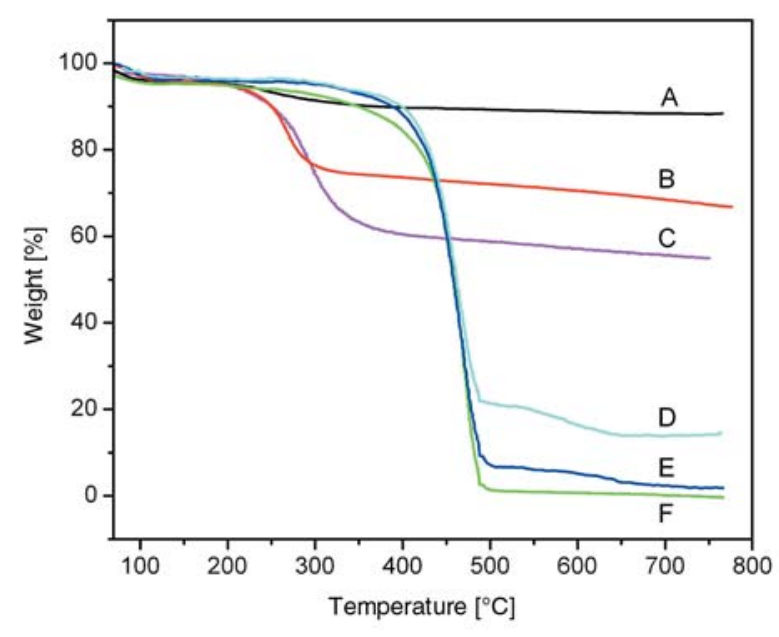

Figure 3. Thermogravimetric analysis data for (A) pristine silica, (B) $\mathrm{SiO}_{2}-\mathrm{NCO},(\mathrm{C})$ caprolactam-functionalized silica, (D and E) PA6/silica nanocomposites with different silica weight proportion, and (F) pure PA6, acquired under nitrogen at a ramp of $5^{\circ} \mathrm{C} / \mathrm{min}$

$68 \mathrm{wt} \%$. This result indicates that the isocyanate graft density, estimated from its $32 \mathrm{wt} \%$ mass loss, is about $2.5 \mathrm{mmol}$ per gram neat silica. Considering the starting silica have a hydroxyl group concentration of $15 \mathrm{wt} \%$, the TGA results indicate that about $33 \%$ of the available hydroxyl groups reacted with TDI despite the insolubility of silica. Supporting evidence comes from the titration experiment, indicating that the functional groups introduced is around $2.2 \mathrm{mmol}$ per gram neat silica. The difference of the graft density between the two methods may lie in the poor dispersibility of $\mathrm{SiO}_{2}-\mathrm{NCO}$ in toluene, which leads to uncompleted reaction between the isocyanate groups and amine. Similarly, the fraction of the caprolactam-functionalized silica sample decomposing below $700^{\circ} \mathrm{C}$ is around $44 \mathrm{wt} \%$, suggesting that almost $100 \%$ of the isocyanate groups reacted with $\varepsilon$-caprolactam. These results are in good agreement with the FTIR analysis. Upon polymerization, the mass loss of nanocomposites could also be calculated from the data in Figure 3, indicating a polymer content of 86 and $98 \%$ for 2 different products.

To gain a more quantitative picture of the extent of silica functionalization, we tried to remove the physically absorbed PA6 chains from the silica. Thus a vial charged with PA6/silica nanocomposites $(50 \mathrm{mg})$ and formic acid $(100 \mathrm{ml})$ was sonicated for $10 \mathrm{~min}$. Then the vial was centrifuged at $10000 \mathrm{rpm}$ for $30 \mathrm{~min}$ and subsequently allowed to stand undisturbed overnight. The supernatant was 


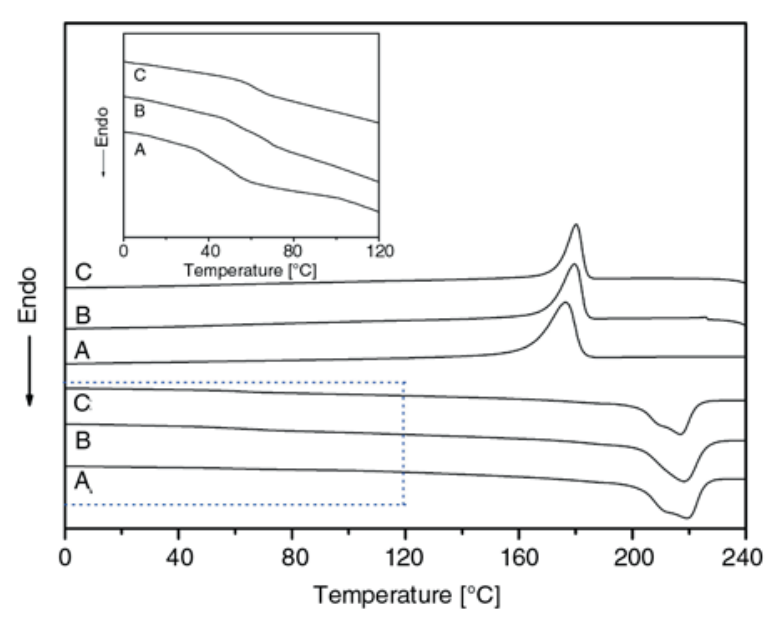

Figure 4. DSC traces of (A) pure PA6, (B and C) PA6/silica nanocomposites with silica contents of 2 and $4 \mathrm{wt} \%$, respectively, acquired under nitrogen at a ramp of $20^{\circ} \mathrm{C} / \mathrm{min}$

carefully separated. This procedure was repeated until the supernatant was free of polymer as determined by precipitating in water. TGA results indicated that the amount of adsorbed PA6 was less than $10 \mathrm{wt} \%$.

Figure 4 illustrates the differential scanning calorimetry (DSC) traces for bulk PA6 and PA6/ silica nanocomposites prepared using the silica initiator. The pure PA6, extracted from the composites with silica content of $2 \mathrm{wt} \%$, shows a glass transition temperature $\left(T_{g}\right)$ at around $59^{\circ} \mathrm{C}$. For the nanocomposites, it was found that the $T_{g}$ of the polymer increased from $59^{\circ} \mathrm{C}$ in the bulk to ca. $67^{\circ} \mathrm{C}$ in the composites with the silica contents of 2 and $4 \mathrm{wt} \%$. This phenomenon can be explained by the attachment of PA6 chains to the silica surface thus imposing constraints over their mobility, resulting in the increase in $T_{g}$. A similar phenomenon has been observed for silica surface grafted polystyrene [29]. Moreover, the presence of the nanosilica in the PA6/silica nanocomposites affects the crystallization of the PA6. The crystallization temperature $\left(T_{c}\right)$ of the PA6 increases from $176^{\circ} \mathrm{C}$ in the bulk to ca. $180^{\circ} \mathrm{C}$ in the composites with the silica content of $4 \mathrm{wt} \%$, which can be attributed to the nucleation effect of nanoparticles although this effect is less profound because the grafting polymers shield the nanoparticles from the direct contacts with PA6 [30]. On the other hand, the melting point $\left(T_{m}\right)$ of the PA6 decreased slightly with increasing the silica contents. Similarly, the melting enthalpy decreased significantly by the presence of nanosilica. This shift can also be attributed to the fact that the polyamide chains are anchored to the silica surface thus reducing the PA6 chains mobility.

The successful implementation of the ring-opening anionic polymerization on the silica surface prompted us to investigate the effects of reaction time and the feed ratio of monomer to silica initiator $\left(R_{f e e d}\right)$ on the monomer conversion and the polymer molecular weight. The monomer conversion [wt $\%$ ] was determined gravimetrically, while the polymer molecular weight was determined using Ubbelohde viscometer. The measurement was done on the material from which $\mathrm{SiO}_{2}$ was removed. For example, $100 \mathrm{mg}$ of the nanocomposite powder was etched with $50 \mathrm{ml}$ of $20 \% \mathrm{HF}$ acid at room temperature for $72 \mathrm{~h}$; the mixture was then diluted with a $200 \mathrm{ml}$ of water and filtered. The obtained polymer was washed thoroughly with water and dried under vacuum at $70^{\circ} \mathrm{C}$ overnight. The recovered polymer was redissolved in formic acid for viscosity measurement. The viscosity-average molecular weight $\left(M_{v}\right)$ of PA6 was calculated from the intrinsic viscosity according to the Equation (2):

$[\eta]=K M_{v}^{\alpha}$

where $K$ and $\alpha$ are constants and are $7.62 \cdot 10^{-2}$ and 0.7 [31], respectively.

We first investigated the effect of reaction time on monomer conversion and polymer viscosity-average molecular weight. Monomer conversion determination was conducted on a series of silica initiator initiated polymerizations that ranged from 2 to $10 \mathrm{~h}$ in duration. At the same time, the molecular weights of these samples were also determined by the method as mentioned above. Figure 5 shows the relationship between reaction time and monomer conversion alongside molecular weight of the resulted PA6. The results reveal that the monomer conversion increases with polymerization time and limited increases are reached after $6 \mathrm{~h}$. This is further supported by the almost linear increase in molecular weight with increasing polymerization time. This result is reasonable because this type of polymerization has been characterized as an equilibrium reaction in that there is a definite temperature dependent concentration of residual monomer and there is no known termination reaction other than that which can occur with impurities [32, 33]. 


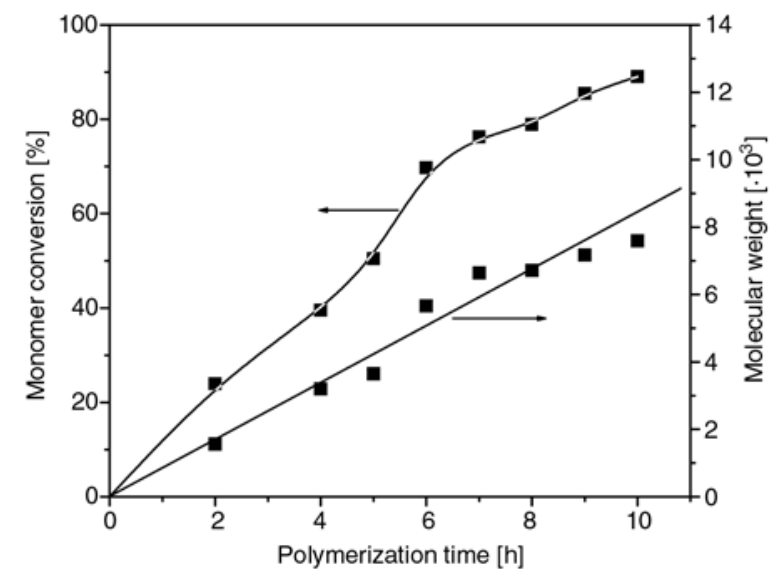

Figure 5. The relationship between polymerization time and the monomer conversion alongside the polymer molecular weight. Conditions, [monomer]: [initiator]:[sodium] = 90:1:6 [mol $/ \mathrm{mol}]$; temperature, $170^{\circ} \mathrm{C}$

In an attempt to control polymer molecular weight, experiments were carried out with variable feed ratio of $\varepsilon$-caprolactam to silica-bound acyl caprolactam initiator $\left(R_{\text {feed }}\right)$ but controlling the monomer conversion at around $80 \mathrm{wt} \%$. Figure 6 shows the relationship between $R_{\text {feed }}$ and molecular weight of PA6 in the composites. It was found that the molecular weight of PA6 could be controlled when the $R_{\text {feed }}$ ranged from 50 to 300 . The molecular weight of PA6 increased almost linear with increasing Rfeed. It should be noted that, in all of these experiments, degassing and dehydration of the reagent mixture at the beginning of each reaction were critical to the polymerization.

High-resolution TEM analysis of bare silica and the PA6/silica nanocomposites was performed by placing a single drop of the formic acid dispersion onto a holey carbon-coated copper grid. Figure 7 depicts representative TEM images for the bare silica particles and PA6/silica nanocomposites. The bare silica particles do not appear as single entities in this dispersion, but tend to form aggregates with an average size of several hundred nanometers although sonication is exerted to the dispersion before testing (Figure 7, A and B). In Figure 7A and B no isolated bare silica particles can be found. In contrast, silica particles in the nanocomposites are observed to have fine dispersion, and the silica particle sizes ranging from 20 to $40 \mathrm{~nm}$ in diameter and with a relatively narrow size distribution. There is no sign of extensive particle agglomeration (Figure 7, C-F). To observe the particles clearly, magnified TEM images for low silica content $(2 \mathrm{wt} \%)$ are

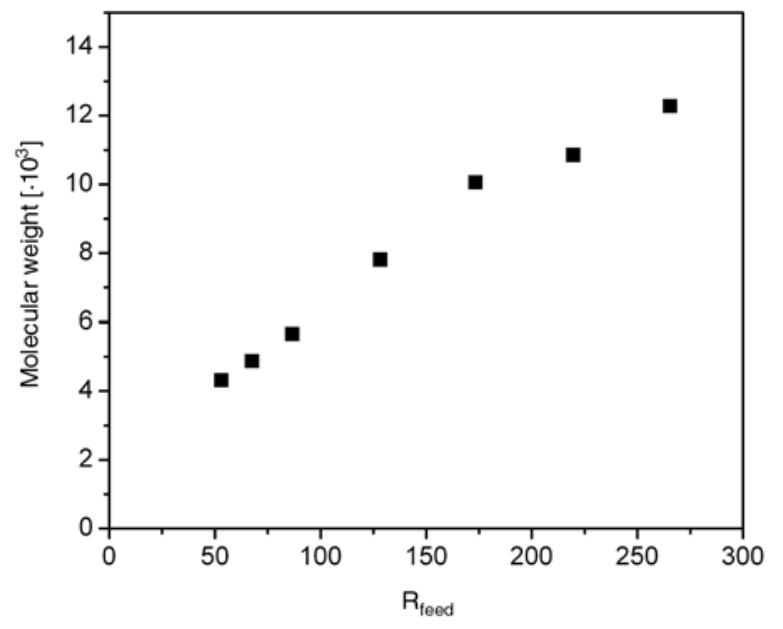

Figure 6. The relationship between $R_{\text {feed }}$ and molecular weight of PA6 in the composites. Conditions, [initiator]:[sodium] = 1:6 [mol/mol]; temperature, $170^{\circ} \mathrm{C}$

shown in Figure 7C and D. In this case, irregular silica particles with sizes ranging from 4 to $20 \mathrm{~nm}$ are found, showing a relatively broad distribution. Conversely, fine spherical particles with ca. $40 \mathrm{~nm}$ in diameter are observed when the silica content is above $10 \mathrm{wt} \%$ (Figure 7, E and F). According to these observations, it is reasonable assumed that the as-received microporous silica particles are aggregates of 'elementary particles' with a diameter of about $4 \mathrm{~nm}$, which is evidenced by the appearance of the clearly discernable 'elementary particles' as shown in Figure 7C and D. Upon mixing the asreceived silica particles with monomer, the $\varepsilon$-caprolactam molecules can enter the interior of the micropores due to interactions between hydroxyl group of silica and amide group of the monomer alongside the high surface to volume ratio of the particles. Thus the polymerization process can create polymer/silica nanocomposites with either an inserted hybrid structure or a deaggregated hybrid structure, which are similar to the intercalated or delaminated hybrid structure in polymer layered silicate nanocomposites as shown in Figure 8. The inserted structure which forms when the silica mass fraction is greater than $10 \mathrm{wt} \%$ is characterized by a fine spherical particle with larger diameter compared to the starting particles, due to polymer entering the interior of the pores (Figure 7, E and F). The deaggregated hybrid structure, which forms when the silica mass fraction is lower than $5 \mathrm{wt} \%$, contains the deformed particles dispersed in the polymeric matrix, their size distribution is relatively broad. 

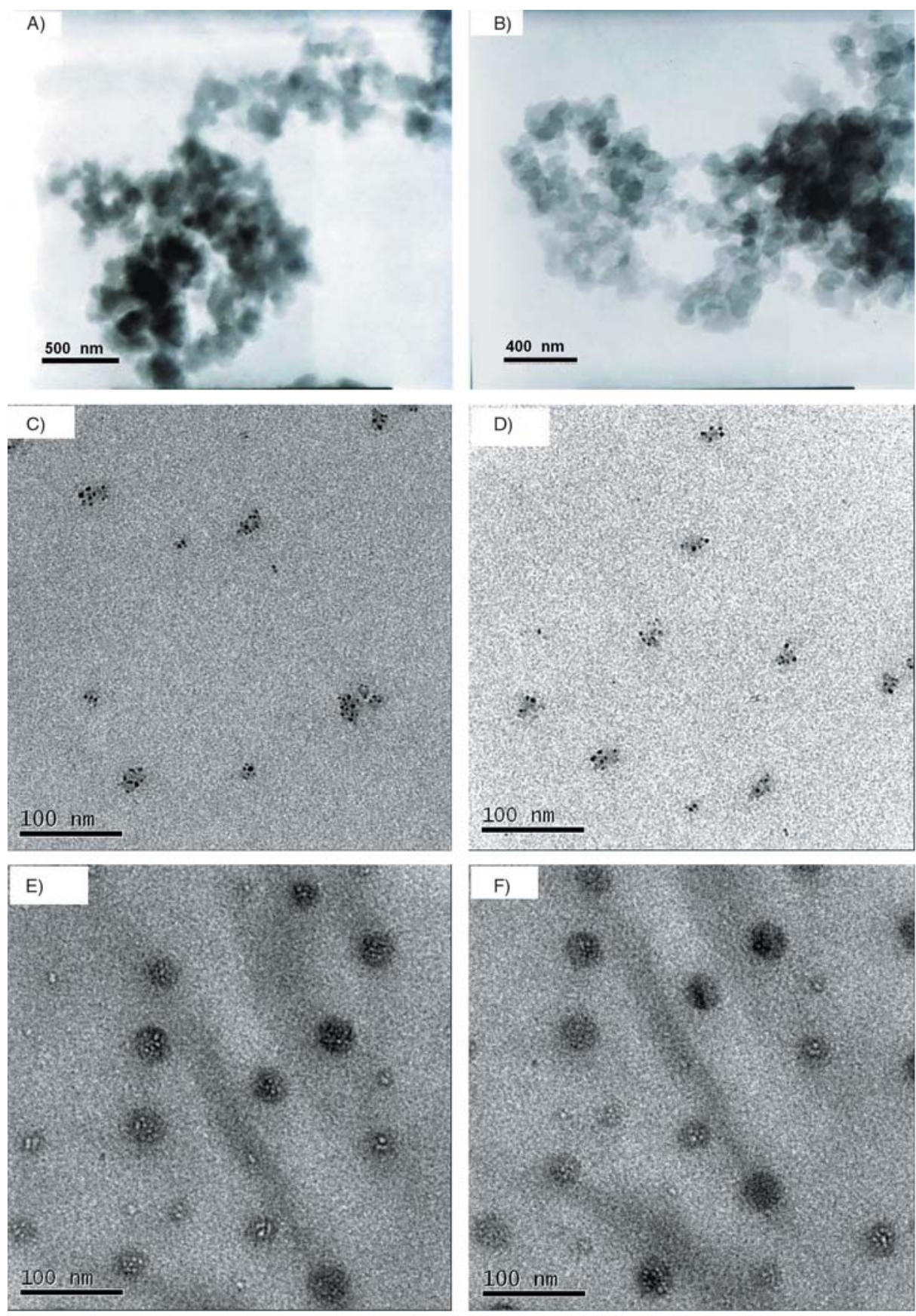

Figure 7. Representative TEM images of pristine silica (A and B), and PA6/silica nanocomposites with different silica mass fraction, $2 \mathrm{wt} \%(\mathrm{C}$ and $\mathrm{D})$, and $10 \mathrm{wt} \%$ (E and F), respectively

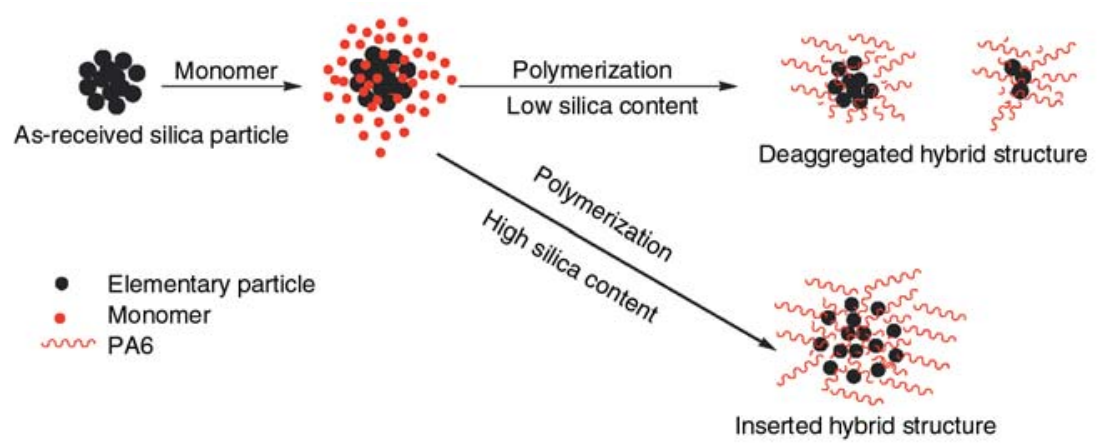

Figure 8. Process used to prepare PA6/silica nanocomposites with either an inserted hybrid structure or a deaggregated hybrid structure 


\section{Conclusions}

We have demonstrated a highly efficient approach to the preparation of PA6/silica nanocomposites using the grafting-from strategy in a two-step process. The formation of the acyl caprolactam initiator by the addition of $\varepsilon$-caprolactam to silica surface isocyanate groups was the first step. The second step was the silica-bound acyl caprolactam initiated 'activated monomer polymerization', allowing for the formation of polymer covalently modified silica nanocomposites. FTIR and TGA analysis indicated that both silica and polymers were present within the material isolated from the polymerization reaction, indicating that they are covalently bound. Analysis of these structures by TEM provided further evidence for the formation of polymerized silica particles. Additionally, it was found that the grafted polymer molecular weight could be adjusted by the feed ratios of monomer to silica initiator.

\section{Acknowledgements}

Financial support for this work was provided by the Scientific Research Funds of Hunan Provincial Education Department (05C081).

\section{References}

[1] Wei Y., Jin D., Yang C., Kels M. C., Qiu K.: Organicinorganic hybrid materials: relations of thermal and mechanical properties with structures. Materials Science and Engineering: Part C, 6, 91-98 (1998).

[2] García M., van Vliet G., Cate M. G. J., Chávez F., Norder B., Kooi B., van Zyl W. E., Verweij, H., Blank D. H. A.: Large-scale extrusion processing and characterization of hybrid nylon-6/SiO 2 nanocomposites. Polymers for Advanced Technologies, 15, 164-172 (2004).

[3] Merkel T. C., He Z., Pinnau I., Freeman B. D., Meakin P., Hill A. J.: Effect of nanoparticles on gas sorption and transport in poly(1-trimethylsilyl-1-propyne). Macromolecules, 36, 6844-6855 (2003).

[4] Merkel T. C., Freeman B. D., Spntak R. J., He Z., Pinnau I., Meakin P., Hill A. J.: Sorption, transport, and structural evidence for enhanced free volume in poly(4-methyl-2-pentyne)/fumed silica nanocomposite membranes. Chemistry of Materials, 15, 109-123 (2003).

[5] Merel T. C., Toy L. G., Andrady A. L., Gracz H., Stejskal E. O.: Investigation of enhanced free volume in nanosilica-filled poly(1-trimethylsilyl-1-propyne) by ${ }^{129} \mathrm{Xe}$ NMR spectroscopy. Macromolecules, 36, 353358 (2003).
[6] Laoutid F., Ferry L., Leroy E., Cuesta J. M. L.: Intumescent mineral fire retardant systems in ethylenevinyl acetate copolymer: Effect of silica particles on char cohesion. Polymer Degradation and Stability, 91, 2140-2145 (2006).

[7] Ji X., Hampsey J. E., Hu Q., He J., Yang Z., Lu Y.: Mesoporous silica-reinforced polymer nanocomposites. Chemistry of Materials, 15, 3656-3662 (2003).

[8] Bartholome C., Beyou E., Bourgeat-Lami E., Chaumont P., Zydowicz N.: Nitroxide-mediated polymerizations from silica nanoparticle surfaces: 'graft from' polymerization of styrene using a triethoxysilyl-terminated alkoxyamine initiator. Macromolecules, 36, 7946-7952 (2003).

[9] Piech M., Bell N. S.: Controlled synthesis of photochromic polymer brushes by atom transfer radical polymerization. Macromolecules, 39, 915-922 (2006).

[10] Ueda J., Sato S., Tsunokawa A., Yamauchi T., Tsubokawa N.: Scale-up synthesis of vinyl polymergrafted nano-sized silica by radical polymerization of vinyl monomers initiated by surface initiating groups in the solvent-free dry-system. European Polymer Journal, 41, 193-200 (2005).

[11] Tsubokawa N., Kogure A., Maruyama K., Sone Y., Shimomura M.: Graft polymerization of vinyl monomers from inorganic ultrafine particles initiated by azo groups introduced onto the surface. Polymer Journal, 22, 827-833 (1990).

[12] Shirai Y., Tsubokawa N.: Grafting of polymers onto ultrafine inorganic particle surface: graft polymerization of vinyl monomers initiated by the system consisting of trichloroacetyl groups on the surface and molybdenum hexacarbonyl. Reactive and Functional Polymers, 32, 153-160 (1997).

[13] Wang Y-P., Pei X-W., He X-Y., Yuan K.: Synthesis of well-defined, polymer-grafted silica nanoparticles via reverse ATRP. European Polymer Journal, 41, 1326-1332 (2005).

[14] Wang Y. P., Pei X. W., Yuan K.: Reverse ATRP grafting from silica surface to prepare well-defined organic/inorganic hybrid nanocomposite. Materials Letters, 59, 520-523 (2005).

[15] Liu P., Liu W. M., Xue Q. J.: In situ radical transfer addition polymerization of styrene from silica nanoparticles. European Polymer Journal, 40, 267-271 (2004).

[16] Hasan M. M., Zhou Y. X., Mahfuz H., Jeelani S.: Effect of $\mathrm{SiO}_{2}$ nanoparticle on thermal and tensile behavior of nylon-6. Material Science and Engineering: A, 429, 181-188 (2006).

[17] Li Y., Yu J., Guo Z. X.: The influence of interphase on nylon-6/nano- $\mathrm{SiO}_{2}$ composite materials obtained from in situ polymerization. Polymer International, 52, 981-986 (2003).

[18] Che J. F., Xiao Y. H., Wang X., Pan A. B., Yuan W., Wu X. D.: Grafting polymerization of polyacetal onto nano-silica surface via bridging isocyanate. Surface and coating technology, 201, 4578-4584 (2007). 
[19] Arnold R. G., Nelson J. A., Verbanc J. J.: Recent advances in isocyanate chemistry. Chemical Reviews, 57, 47-76 (1957).

[20] Simons D. M., Arnold R. G.: Relative reactivity of the isocyanate groups in toluene-2,4-diisocyanate. Journal of the American Chemical Society, 78, 1658-1659 (1956).

[21] Sumitomo H., Hashimoto K.: Polymerization of bicyclic oxalactam. A novel polyamide poly(tetrahydropyran-2,6-diyliminocarbonyl). Macromolecules, 10, 1327-1331 (1997).

[22] Hu G. H., Cartier H., Plummer C.: Reactive extrusion: Toward nanoblends. Macromolecules, 32, 4713-4718 (1999).

[23] Bertoldo M., Cappelli C., Caranorchi S., Liuzzo V., Bronco S.: Understanding the accelerating effect of $\varepsilon$ caprolactam on the formation of urethane linkages. Macromolecules, 38, 1385-1394 (2005).

[24] Li R. H., Barbari T. A.: Protein-transport through membranes based on toluene diisocyanate surfacemodified poly(vinyl alcohol) gels. Journal of Membrane Science, 88, 115-125 (1994).

[25] Blitz P., Little C. B.: Fundamental and applied aspects of chemically modified surfaces. The Royal Society of Chemistry, Cambridge (1999).

[26] Wang L. W., Sigmund W., Aldinger K.: A systematic approach for dispersion of silicon nitride powder in organic media: ii, dispersion of the powder. Journal of the American Ceramic Society, 83, 697-702 (2000).
[27] Boven G., Oosterling M. L. C. M., Challa G., Schonten A. J.: Grafting kinetics of poly(methylmethacrylate) on microparticulate silica. Polymer, 31, 2377 2383 (1990).

[28] Shylesh S., Sharma S., Mirajkar S. P., Singh A. P.: Silica functionalised sulphonic acid groups: synthesis, characterization and catalytic activity in acetalization and acetylation reactions. Journal of Molecular Catalysis A: Chemical, 212, 219-228 (2004).

[29] Sertchook H., Avnir D.: Submicron silica/polystyrene composite particles prepared by a one-step sol-gel process. Chemistry of Materials, 15, 1690-1694 (2003).

[30] Reynaud E., Jouen T., Ganthier C., Vigier G., Varlet J.: Nanofillers in polymeric matrix: a study on silica reinforced PA6. Polymer, 42, 8759-8768 (2001).

[31] Brandrup J., Immergut E. H.: Polymer Handbook. John Wiley and Sons, New York (1975).

[32] Korshak V. V., Frunze T. M., Zaitsev V. I., Kurashev V. V., Babchinitser T. M.: Anionic polymerization of $\varepsilon$-caprolactam in the presence of activators containing unsaturated groups. Polymer Science U. S. S. R, 12, 475-484 (1970).

[33] Mikheyev V. N.: Polymer molecular weight in anionic polymerization of caprolactam Polymer. Polymer Science U.S.S.R, 15, 1829-1834 (1973). 